\title{
Wie viel verdienen CFOs und Controller 2014?
}

Die Höhe des Gehalts ist enorm wichtig, um qualifizierte Mitarbeiter $\mathrm{zu}$ finden und im Unternehmen zu halten. Zudem trägt das Gehalt entscheidend zur Zufriedenheit der Mitarbeiter bei, drückt es doch die Anerkennung des Arbeitgebers aus. Zu diesen Ergebnissen kommt die von dem Personaldienstleistungsunternehmen Robert Half in Auftrag gegebene „Workplace Survey 2013“, für die 200 HR-Manager befragt wurden.

Welche Gehälter im Finanz- und Rechnungswesen 2014 gezahlt werden, hat der Personaldienstleister in einer Übersicht zusammengestellt. Im Durchschnitt verdienen im Jahr 2014:

\section{Controller}

- mit drei bis fünf Jahren Berufserfahrung 44.000 bis 64.000 Euro

- mit sechs bis neun Jahren Berufserfahrung 62.000 bis 75.000 Euro

\section{Senior Controller}

- mit sechs bis neun Jahren Berufserfahrung 65.000 bis 81.000 Euro

- mit zehn bis 15 Jahren Berufserfahrung 78.000 bis 100.000 Euro

- mit mehr als 15 Jahren Berufserfahrung mehr als 95.000 Euro

\section{Leiter Controlling}

- mit drei bis fünf Jahren Berufserfahrung 70.000 bis 80.000 Euro

- mit sechs bis neun Jahren Berufserfahrung 79.000 bis 94.000 Euro

- mit zehn bis 15 Jahren Berufserfahrung 92.000 bis 107.000 Euro

- mit mehr als 15 Jahren Berufserfahrung mehr als 105.000 Euro

Der Studie zufolge gibt es aber auch große regionale Unterschiede. Die höchsten Gehälter werden demnach für Finanzspezialisten in Frankfurt am Main gezahlt. Dort können Controller mit rund $14 \%$ mehr Gehalt als der Durchschnitt rechnen, gefolgt von Stuttgart und München (mit je $11 \%$ ).

\section{Mehr zum Thema}

Pfennig, C.: Der Kampf um Top-Controller geht in die nächste Runde - Was können CFOs tun?, in: Controlling \& Management Review, Jg. 57 (2013), Sonderheft 1, S. 96 - 100, SiP $^{*}$ www.springerprofessional.de/4411884

Sylvia Meier, Vera Treitschke, Wiesbaden

\section{Steuerabteilungen bekommen neue Aufgaben}

Die Aufgabenbereiche der Steuerabteilungen in Unternehmen verändern sich. Dies zeigen die Ergebnisse der Studie „European Tax Survey - The Benefits of Stability“ der internationalen Wirtschaftsprüfungsgesellschaft Deloitte. Befragt wurden europaweit 1.000 große Unternehmen und Konzerne. Für Deutschland zeigt sich, dass von Steuerabteilungen ein immer tieferes Verständnis für Unternehmensprozesse erwartet wird.

Führungskräfte in der Steuerabteilung verwenden laut der Umfrage die meiste Zeit für Organisationstätigkeiten, die Zusammenarbeit mit anderen Abteilungen und Projektmanagement außerhalb des Steuerbereichs. Interessanterweise wenden die Steuerabteilungsleiter auch weniger Zeit für steuerrechtliche Fragen, den Kontakt zur Steuerbehörde und die Darstellung steuerrechtlicher Themen auf.
Ihren Beitrag zur Wettbewerbsfähigkeit des Unternehmens leistet eine Steuerabteilung, wenn sie Steuerdeklarations- und Dokumentationsanforderungen fristgerecht einhält. Das meinen $70 \%$ der Befragten. Doch auch die enge Einbindung der Abteilung in die Unternehmensstrategie wird immer wichtiger. Mitarbeiter der Steuerabteilung müssen die Unternehmenssteuerstrategie aktiv mitgestalten und das Thema Steuerreporting verstärken.

Mehr zum Thema

Risse, R.: Steuercontrolling- und Reporting, Wiesbaden 2010, SfP $^{*}$ www.springerprofessional.de/1833818

Sylvia Meier, Wiesbaden 